\title{
Asymmetric $\Delta E$ Effect and its Use as a Magnetic Field Sensor
}

\author{
S. Atalay*, V.S. Kolat, and T. Izgi \\ Physics Department, Faculty of Science, Inonu University, Malatya, Turkey \\ (Received July 16, 2019; revised version July 25, 2019; in final form July 25, 2019)
}

The $\Delta E$ effect was studied using $\mathrm{Fe}_{77} \mathrm{~B}_{16} \mathrm{Si}_{5} \mathrm{Cr}_{2}$ amorphous ribbon, and asymmetry in the $\Delta E$ curves was created using micro magnets placed on the surface of the ribbon. Asymmetry was also observed in the vibration amplitude versus the magnetic field, and magnetization loops of amorphous ribbons with micro magnets were observed. The results show that the asymmetries in the $\Delta E$ curves and magnetisation loops are in agreement with each other. A linear change in vibration amplitude as a function of the applied magnetic field in the $\pm 100 \mathrm{~A} / \mathrm{m}$ region was observed, which is important for sensor applications.

DOI: 10.12693/APhysPolA.136.531

PACS/topics: Asymmetry, $\Delta E$ effect, Young's modulus

\section{Introduction}

The $\Delta E$ effect can be defined as a variation of Young's modulus as a function of the magnetic field, and has been intensively studied in amorphous ribbons [1-4], wires [5-7], and films [8], since changes in modulus or resonant frequency have many technological applications [9-13]. The $\Delta E$ effect in amorphous alloys is much larger than that in crystalline materials. Due to the lack of crystal anisotropy in amorphous alloys, the change in the Young's modulus as a function of the magnetic field can reach nearly $80 \%$ [6].

Berry and Prichet [14], Spano et al. [15], Livingston [16], Squire [17], Kouzoudis and Nikolais [18] and Dapino et al. [19] have tried to model the $\Delta E$ effect. Livingston proposed a simple model based on the rotation of magnetic moments. This model considers an amorphous ribbon which is annealed in a magnetic field parallel to the ribbon width, producing a magnetic easy axis that is perpendicular to the ribbon length. In this model, the magnetic field dependence of Young's modulus is given by the expression:

$$
\frac{E(H)}{E_{S}}=\frac{1}{1+9\left(\lambda_{S}^{2} E_{S} H^{2}\right) /\left(\mu_{0}, M_{S}, H_{k}^{3}\right)},
$$

where $H$ is the applied magnetic field, $\lambda_{S}$ is the saturation magnetization, $E_{S}$ is the modulus at saturation, $H_{k}$ is the anisotropy field given by $\frac{2 K}{\mu_{0} M_{S}}, M_{S}$ is the saturation magnetization, and $K$ is the total anisotropy constant. According to Livingston's model, a large $\Delta E$ effect can be obtained with high $\lambda_{S}$ and $M_{S}$ and low $K$. All these parameters vary with the composition of the alloy, and in particular, the value of $K$ is very sensitive to annealing temperature and time.

The Young's modulus of value around zero changes gradually, and the vibration amplitude also goes to zero in a field of value $H \approx 0 \mathrm{~A} / \mathrm{m}$, leading to problems in terms of practical applications.

*corresponding author; e-mail: selcuk.atalay@inonu.edu.tr
Similar problems in sensor design have been reported based on different measurement techniques. For example, the magnetoimpedance sensor output changes gradually in a field of value $H \approx 0 \mathrm{~A} / \mathrm{m}$, and the magnetoimpedance curve is symmetric for both positive and negative magnetic field values [20]. A sharp, linear variation in output is desirable for sensor applications. An asymmetric curve has been proposed as a possible solution for obtaining a sharp, linear change in a magnetic field of value around zero [20]. In this study, we report an asymmetric $\Delta E$ curve for the first time in the literature.

\section{Experimental}

It has been reported that $2605 \mathrm{~S} 3 \mathrm{~A}\left(\mathrm{Fe}_{77} \mathrm{~B}_{16} \mathrm{Si}_{5} \mathrm{Cr}_{2}\right)$ amorphous ribbon shows a good magnetoelastic response, because it has a high magnetostriction of $20 \times 10^{-6}$. Amorphous ferromagnetic 2605S3A ribbons were therefore used in our measurements of the $\Delta E$ effect. Samples with a thickness of $25 \mu \mathrm{m}$ were cut to $40 \mathrm{~mm}$ in length and $1 \mathrm{~mm}$ in width, and were then annealed at $450^{\circ} \mathrm{C}$ in air for various periods. The samples were cleaned with acetone and alcohol before measurements of $\Delta E$ were carried out.

The magnetic field dependence of the Young's modulus was measured using a resonance/antiresonance technique. The measurement system used was similar to previous studies [21, 22]. An AC magnetic field of $10 \mathrm{~A} / \mathrm{m}$ was applied along the sample length by the driver coil, leading to longitudinal vibration of the ribbon and inducing voltages in the pick-up coil. Details of the measurement system are given in [23]. The variation in signal amplitude as a function of frequency at a magnetic field of $280 \mathrm{~A} / \mathrm{m}$ is presented in Fig. 1. The resonance frequency, $f$, was taken to be the maximum of this curve. Since $E$ is proportional to $f^{2}$ and the ratio of the squares of resonant frequencies is equal to the ratio of the moduli under different applied fields [7, 14]:

$$
\frac{E(H)}{E_{S}}=\left(\frac{f(H)}{f_{S}}\right)^{2}
$$


where $f(H)$ and $f_{S}$ are the resonant frequencies for an applied field $H$, and in the saturated state, respectively. In this way, the relative $\Delta E$ was obtained with respect to $H$ using Eq.(2). The magnetic field was changed, and the resonance frequency and its amplitude were measured for a series of field strengths $H$, as it cycled from a maximum in one direction to a maximum in the opposite direction. Hysteresis loops of samples were measured at DC using a fully automatized experimental set-up. The surface crystallization process was monitored using a LEO EVO 40VP scanning electron microscope (SEM) and energy dispersive X-ray (EDX).

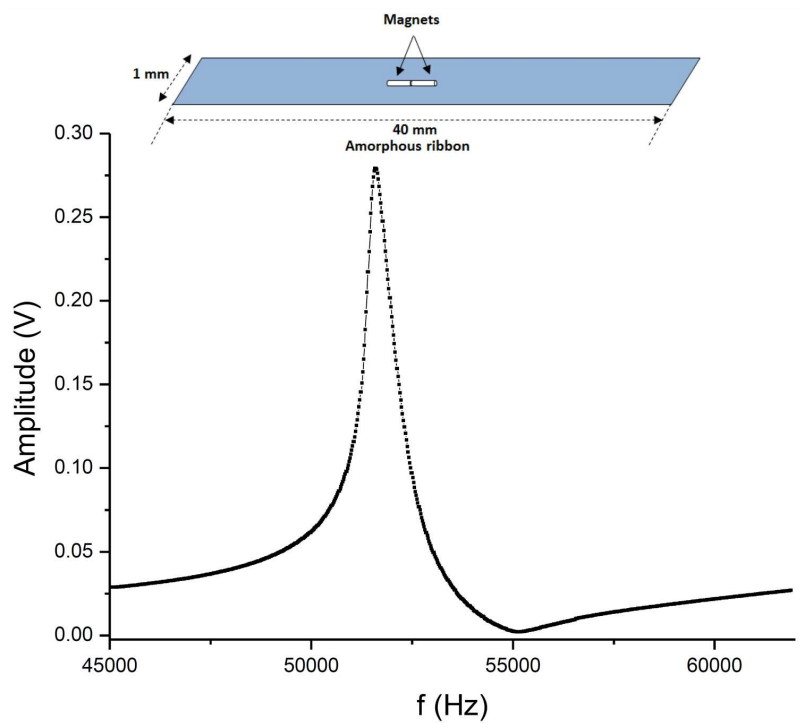

Fig. 1. Variation in signal amplitude as a function of frequency at $280 \mathrm{~A} / \mathrm{m}$ for ribbon annealed for $120 \mathrm{~min}$. The inset shows sample dimensions and magnet positions.

$\Delta \mathrm{E}$ and hysteresis loop measurements were made both with and without micro magnets. NdFeB micro magnets were obtained from China, and were $1 \mathrm{~mm}$ in length and $300 \mu \mathrm{m}$ in diameter. The magnets were placed in the middle of the amorphous ribbon, as shown in the inset of Fig.1.

\section{Results and discussion}

SEM investigation showed that samples annealed for up to $10 \mathrm{~min}$ had a smooth surface and no trace of crystallization. In samples annealed for a longer period, small metal-oxide crystallites started to grow and increased with annealing time. It was observed that the surface of a sample annealed for 120 min was nearly full of metaloxide crystallites.

$\Delta E$ curves and resonance frequency amplitude voltage versus magnetic field were measured for samples annealed for 5, 10, 30, 45, 75, 90 and $120 \mathrm{~min}$, and some of these curves are presented in Figs. 2-5. It was found that annealing for short periods (up to $10 \mathrm{~min}$ )
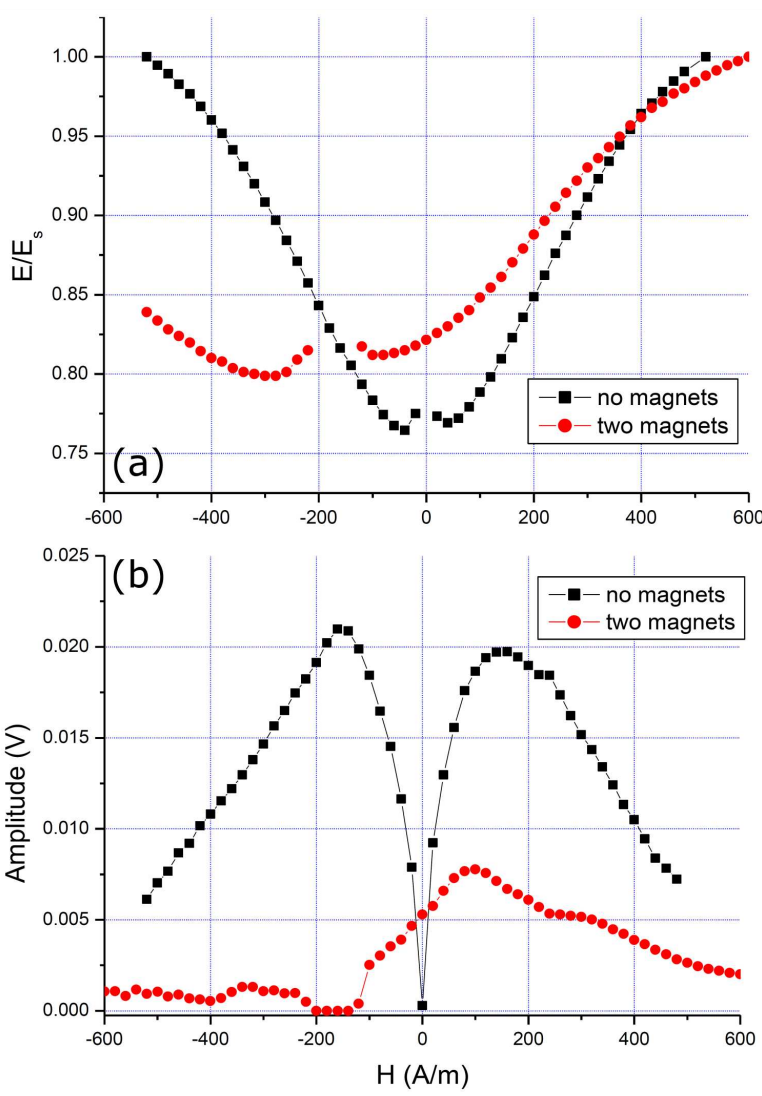

Fig. 2. (a) $\Delta E$ curves for ribbons annealed for $5 \mathrm{~min}$ with two magnets and without magnets. (b) Dependence of vibration amplitude on magnetic field.

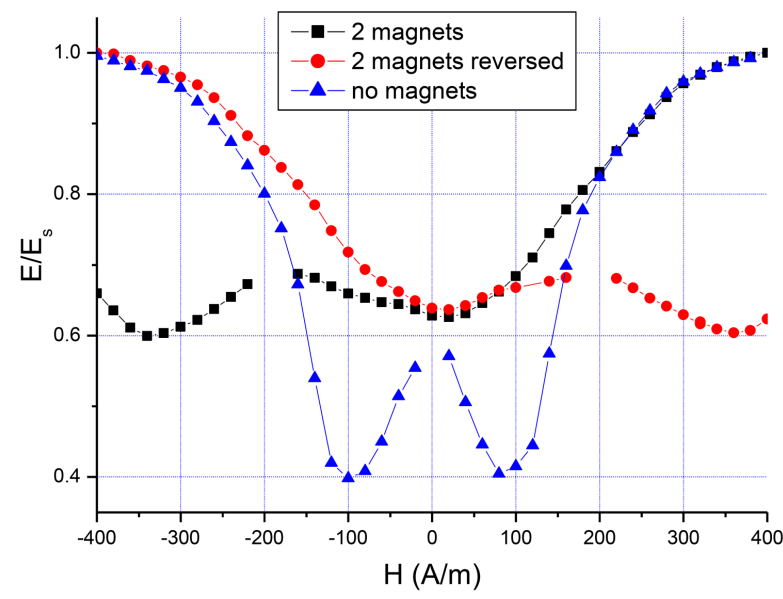

Fig. 3. $\Delta E$ curves for ribbons annealed for $45 \mathrm{~min}$ with no magnets and two magnets.

removed internal stress and therefore reduced magnetoelastic anisotropy, leading to an increase in the magnitude of the $\Delta E$ effect. Longer annealing times induced partial crystallization, which in the case of amorphous ribbon is generally nucleated initially at the surfaces. Slight differences in the composition and density of the crystalline and amorphous phases cause stress fields to be set up between the surfaces, thus inducing a macroscopic 
stress-induced anisotropy along the direction of the ribbon width. For a positively magnetostrictive ribbon, an easy axis forms along the sample width via surface crystallization. This behavior has been observed in many previous studies $[6,24]$. The shape of the $\Delta E$ curve changes from a $V$-shape to a $W$-shape as the relative percentage of partial crystallization increases. This change is due to the variation in the easy axis from the direction of the ribbon length to the perpendicular direction. It was also observed that the vibration amplitude of the sample $\left(V_{a}\right)$ reaches a maximum at around $H_{k}$, and $V_{a}$ decreases with increasing $H$. Most importantly, $V_{a}$ becomes zero in all ribbon samples annealed for a short time, and is nearly zero at $H \approx 0 \mathrm{~A} / \mathrm{m}$ for ribbons annealed for longer times, meaning that the measurement of amplitude and therefore Young's modulus becomes almost impossible. Additionally, it was observed that in all $\Delta E$ curves, the modulus changes gradually at a magnetic field of value around zero. In order to solve this problem, an asymmetry was created in the $\Delta E$ curves by placing two micro magnets in the middle of the ribbon.
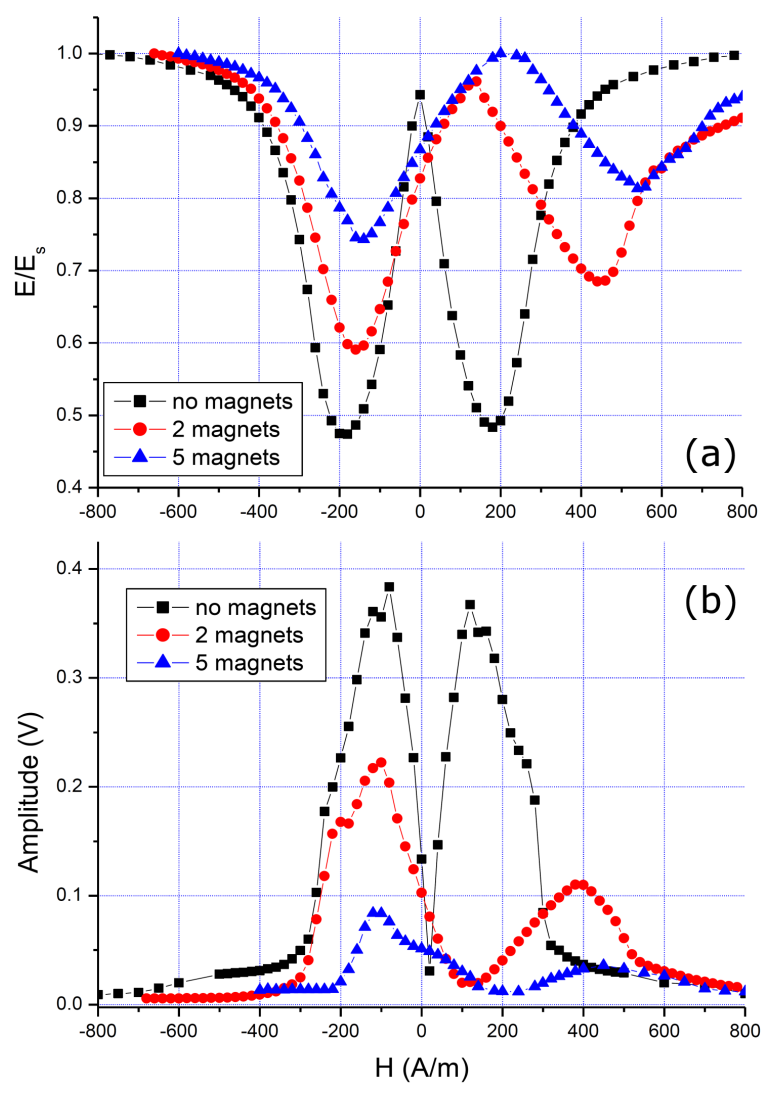

Fig. 4. Effect of the number of magnets on (a) $\Delta E$ curves and (b) $V_{a}-H$ curves for sample annealed for $75 \mathrm{~min}$.

The asymmetric $\Delta E$ and vibration amplitude versus magnetic field $\left(V_{a}-H\right)$ curves are presented in Figs. 2-5. Two magnets were placed in the middle of the ribbon, as shown in the inset of Fig. 1. These results show that
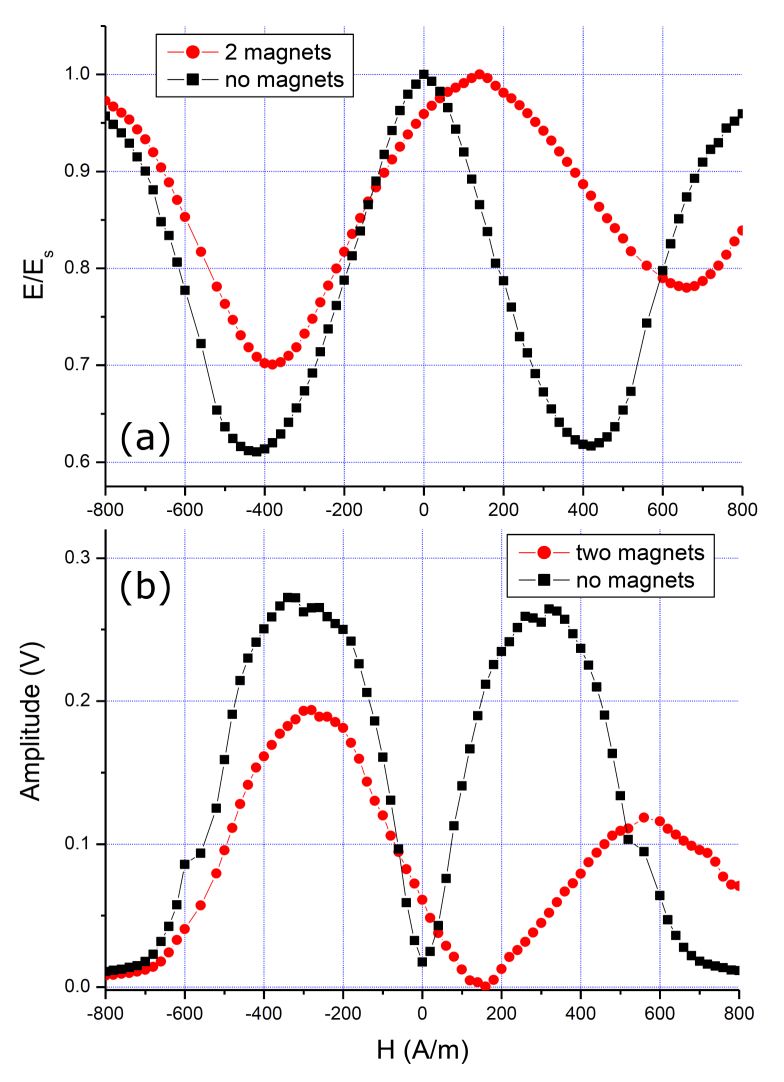

Fig. 5. (a) $\Delta E$ curves for ribbons annealed for $120 \mathrm{~min}$ with two magnets and without magnets. (b) Dependence of the vibration amplitude on magnetic field.

the $\Delta E$ and $V_{a}-H$ curves are not the same in the negative and positive magnetic field regions, and therefore an asymmetry forms in both the $\Delta E$ and $V_{a}-H$ curves.

The sample annealed for $5 \mathrm{~min}$ shows a nearly $V$-shaped $\Delta E$ curve, and the $V_{a}-H$ curve has an inverted $W$-shape. It was found that the general shape of the $\Delta E$ curve remained the same while the $\Delta E$ curve shifted to the negative magnetic field region at about $200 \mathrm{~A} / \mathrm{m}$. Additionally, in this sample we could not measure the Young's modulus at around $-200 \mathrm{~A} / \mathrm{m}$, although the Young's modulus was measured at around $H=0 \mathrm{~A} / \mathrm{m}$ without difficulty (Fig. 2a). The $V_{a}-H$ curve with magnets shows an interesting asymmetry whereby the amplitude goes almost to zero for values of magnetic field lower than $-100 \mathrm{~A} / \mathrm{m}$. The change in $V_{a}$ as a function of magnetic field is nearly linear in the $\pm 100 \mathrm{~A} / \mathrm{m}$ region (Fig. 2b).

The effect of the magnet direction with respect to the direction of the applied field was also investigated (Fig. 3). The asymmetric shape of the $\Delta E$ curve was reversed when the magnetic poles were reversed. The magnetic field was also swept from $\left(-H_{\max }\right)$ to $\left(+H_{\max }\right)$ and back to $\left(-H_{\max }\right)$ for this sample, and we observed almost zero hysteresis in the $\Delta E$ curves.

Figures 4 and 5 show the $\Delta E$ and $V_{a}-H$ curves for ribbons annealed for 75 and $120 \mathrm{~min}$, respectively. The $\Delta E$ and $V_{a}-H$ curves were also measured with magnets for 
wire annealed for $75 \mathrm{~min}$, to investigate the effect of the number of magnets. It should be noted that the directions of the magnets are the opposite of those in the measurements shown in Fig. 2. The $\Delta E$ curves show that $H_{k}$ shifts by about $120 \mathrm{~A} / \mathrm{m}$ towards the positive $H$ direction, and with an increasing number of magnets, this shift reaches a value of about $200 \mathrm{~A} / \mathrm{m}$. The magnitudes of the $\Delta E$ effect and $V_{a}$ also decrease with increasing magnet number.

Figure 6 shows magnetization loops for amorphous ribbons without and with magnets. The results show that the magnets affect the shape of the magnetization loops and that asymmetry also forms in the $M-$ $H$ loop. These results can be explained on the basis of a non-uniform field created by the micro magnets. It was found that magnets have three different effects on the $M-H, \Delta E$, and $V_{a}-H$ curves. A shift in the magnetization and a decrease in the magnitude of the $\Delta E$ and $V_{a}-H$ curves occur depending on the direction of the magnets. The shift in the magnetization axis is probably due to the contribution to magnetization from the magnets. The shift in the magnetic field axis is due to the bias field effect from the magnets, and the magnets induce larger anisotropy along one half of the $M-H, \Delta E$, and $V_{a}-H$ curves depending on the orientation of the magnet. According to Eq.(1), the magnitude of the $\Delta E$ effect is inversely proportional to the total anisotropy constant. The $M-H$ loops clearly show that the sample with magnets also has an asymmetric $K$ on the positive and negative sides of $H$, and therefore the magnitude of the $\Delta E$ effect is higher on one side.

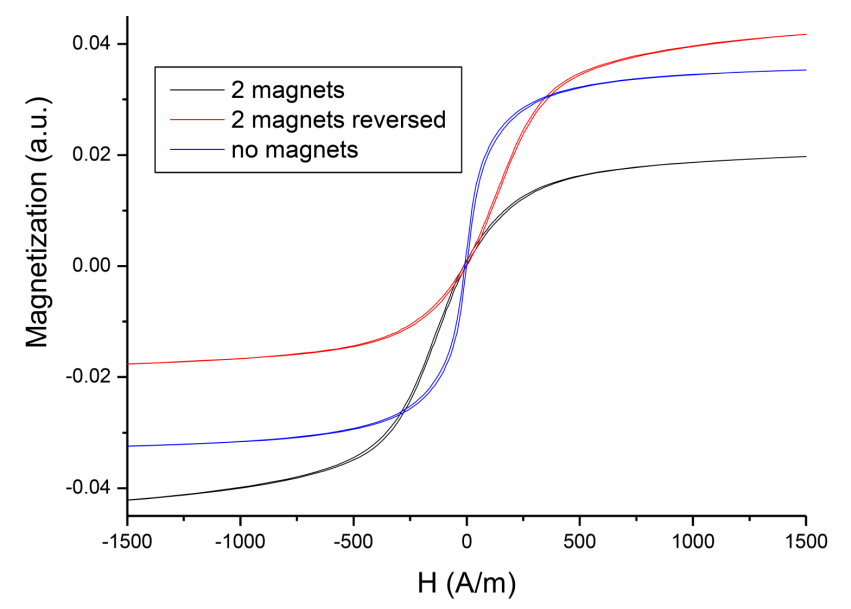

Fig. 6. Magnetization loops for ribbons annealed for 5 min with no magnets (and with two magnets as shown in the inset of Fig. 1) and with reversed magnets (as shown in the inset of Fig. 1).

\section{Conclusions}

A non-uniform field created by micro magnets gives rise to a non-uniform anisotropy in amorphous ferromagnetic 2605S3A ( $\left.\mathrm{Fe}_{77} \mathrm{~B}_{16} \mathrm{Si}_{5} \mathrm{Cr}_{2}\right)$ ribbon. As a result, a non-uniform $M-H$ loop, $\Delta E$ and $V_{a}-H$ curves were obtained. The anisotropy and therefore the asymmetry can be controlled by the number of magnets, i.e., the magnitude of a non-uniform magnetic field. It can be assumed that through the formation of a non-uniform magnetic field, asymmetry can be created in many other magnetic parameters such as magnetostriction, magnetoimpedance, and the magnetoresistance effect.

One of the main problems of magnetoelastic sensors is that the resonance frequency amplitude goes to zero. Our results also show that this can be easily solved by placing micro magnets on top of the sample surface. Moreover, a linear amplitude change as a function of the applied magnetic field in the $\pm 100 \mathrm{~A} / \mathrm{m}$ region was observed for nearly all of the annealed samples. So the sensor can be used to measure magnetic field value for low magnetic field regions.

\section{Acknowledgments}

This work was supported by Inonu University under project number 1854 .

\section{References}

[1] Z. Kaczkowski, Mat. Sci. Eng. 226-228, 614-625 (1997).

[2] A.A. Gavrilyuk, A.L. Semenov, A.R. Gafarov, A.V. Gavrilyuk, N.P. Kovaleva, S.M. Zubritskii, B.V. Gavrilyuk, N.V. Morozova, Russ. Phys. J. 59, 5 (2016).

[3] J. Gutierrez, J.M. Barandiaran, P. Minguez, Z. Kaczkowski, P. Ruuskanen, G. Vlasak, P. Svec, P. Duhaj, Sensors Actuat. A-Phys. 106, 69 (2003).

[4] J.M. Barandiaran, J. Gutierrez, Z. Kaczkowski, D. De Cos, J. Non-Cryst. Solids 329, 43 (2003).

[5] A.A. Gavrilyuk, A.L. Semenov, E.A. Golygin, A.R. Gafarov, A.A. Zinchenko, N.V. Morozova, A.V. Gavrilyuk, A.Yu. Mokhovikov, Phys. Met. Metallogr. 115, 864 (2014).

[6] S. Atalay, P.T. Squire, IEEE Trans. Magn. 28, 3144 (1992).

[7] S. Atalay, P.T. Squire, J. Appl. Phys. 70, 6516 (1991).

[8] A. Ludwing, E. Quandt, IEEE Trans. Magn. 38 2829 (2002).

[9] G. Herzer, Sensors Lett. 5, 259 (2007).

[10] L.G. Puckett, G. Barrett, D. Kouzoudis, C. Grimes, L.G. Bachas, Biosens. Bioelectron. 18, 675 (2003).

[11] D. Kouzoudis, C. A. Grimes, J. Appl. Phys. $\mathbf{8 7}$, 6301 (2000).

[12] R. Guntupalli, J. Hu, R.S. Lakshmanan, T.S. Huang, J.M. Barbaree, B.A. Chin, Biosens. Bioelectron. 22, 1474 (2007).

[13] B. Gojdka, R. Jahns, K. Meurisich, H. Greve, R. Adelung, E. Quandt, R. Knochel, F. Faupel, Appl. Phys. Lett. 99, 3664135 (2011).

[14] B.S. Berry, Scr. Metall. 16, 1407 (1982). 
[15] M.L. Spano, K.B. Hathaway, H.T. Savage, J. Appl. Phys. 53, 2667 (1982).

[16] J.D. Livingston, Phys. State Solids A 70, 591 (1982).

[17] P.T. Squire, J. Magn. Magn. Mater. 87, 299 (1990).

[18] D. Kouzoudis, V. Nikolakis, J. Magn. Magn. Mater. 395, 59 (2015).

[19] M.J. Dapino, R.C. Smith, A.B. Flatau, in: Proc. SPIE 3985, Smart Structures And Integrated Systems, 2000, p. 174.

[20] M. Knobel, M. Vazquez, L. Kraus, M.L. Sanchez, R. Valenzuela A.P. Zhukov, Sensors Actuat. A-Phys. 59, 20-29 (1997).
[21] H.T. Savage, R. Abbundi, A.E. Clark, O.D. McMasters, J. Magn. Magn. Mater. 5, 609-610 (1980).

[22] C.A. Grimes, S.C. Roy, S.J. Rani, Q.Y. Cai, Sensors 11, 2809 (2011).

[23] S. Atalay, V.S. Kolat, N. Bayri, T. Izgi, J. Supercond. Nov. Magn. 29, 1551 (2016).

[24] P.J. Schurer, A.H. Morrish, M.J. Stavn, Phys. Status Solidi A 64, 343 (1981). 